\title{
(CON) TEXTOS Y SILENCIOS: LA COMUNIDAD COMO ESPACIO DE APRENDIZAJE NO FORMAL
}

Óscar Delgado Cascantel

"...les vamos transmitiendo nuestras frustraciones con la leche templada y en cada canción...".

J.M. Serrat

\section{Resumen}

La presente ponencia surge de la experiencia de Jornadas Comunitarias Amor Joven: un modelo de abordaje comunitario de la sexualidad de los $y$ las adolescentes, que pretendía llevar la educación de la sexualidad joven a comunidades de diferentes regiones del país.

Las siguientes son las reflexiones sobre la experiencia en dos niveles: primero, por la decisión de apoyar procesos de aprendizaje y no procesos de enseñanza-aprendizaje, en la educación no formal y, segundo, el asunto de la necesidad de crear sinergias entre los sistemas de aprendizaje.
Palabras claves: educación no formal, sexualidad, comunidad rural, juventud.

\section{Abstract}

This article is the result of a paper presented at the "Youth Love Communitarian Conferences: a communitarian model of youth sexuality", which pretended to teach youth sexual education into different communities and regions of the country.

The author contributes with thoughts about the experience in two different levels. First, the decision of supporting learning processes instead of teaching-learning processes, in informal education; and second, the necessity

1 Licenciado en Antropologia Social de la UCR, cursó estudios de Psicologia en la UNAM. Trabaja como consultor social desde hace 13 años en temas de juventud. juventud rural, educación rural, educación no formal. metodologias de la educación, y diversos proyectos de desarrollo social.

Recibido: 15 de noviembre-2006 - Aprobado: 30 de noviembre-2006 
of creating synergy between learning systems.
Keywords: informal education, sexuality, rural community, youth.

$\mathrm{N}$ uestros sistemas educativos han sido, son, y muy probablemente serán durante un tiempo más, procesos de educación bancaria donde una persona enseña y el resto, aprende. Pensé por un momento, que esta idea un tanto monolítica del proceso educativo, tenía que ver con las ideas de nuestros próceres; sin embargo, me atrevo a decir que tiene un arraigo mucho más lejano y va con la génesis de las ideas de transmisión de conocimientos que estaban en manos de los religiosos que usaban el mismo mecanismo de dominación, usado por la Iglesia en tiempos anteriores: dominar por el adoctrinamiento, y para este fin lo mejor era que uno supiera y otro aprendiera.

Cuando empezamos a trabajar el tema de la sexualidad con los y las docentes y los jóvenes, comprendimos que este sistema no sólo estaba obsoleto, sino que reproducía los mitos y estereotipos que compartían las personas jóvenes y adultas y que, además, las últimas se encargaban de diseminar como semillas en tierra fértil.

Estos asuntos nos llevaron a comprender que nos enfrentábamos a dos realidades: por un lado, que el proceso de enseñanza-aprendizaje no reunía los requisitos para atender la educación en sexualidad y, por otro, que no sólo se tenía que educar a los jóvenes, sino también y tal vez, a los adultos. Esto sería posible con un enfoque de aprendizaje comunitario.

Se debe entender todo proceso educativo en dos dimensiones: la enseñanza y el aprendizaje. Hay que reconocer que los sujetos aprenden sin necesidad de ser enseñados, que las capacidades para aprender son distintas, y que cuando se trata de una educación de la sexualidad, este debe ser un proceso para adquirir habilidades de autocuidado, mutuocuidado, sociocuidado y prevención, para que la toma de decisiones sea desde un lugar de reflexión y responsabilidad (Ministerio de Salud de Brasil, 1999).

Este tipo de educación parte de la posibilidad de darle la palabra al sujeto, de valorar su conocimiento e invitarle a crear a partir de este, uno propio libre de temores, estereotipos y mitos; así, al aprender haciendo, se producen cambios reales en el sentir y en el actuar de las personas.

El tema de brindar información no desvaloriza esta estrategia, pero tampoco la prioriza desde la lógica de un único saber que se ofrece; parte de que los sujetos tienen un saber propio, que este es importante, lo retoma y procura que se construya otro. Esta estrategia de aprendizaje aleja de sus 
intenciones aquellas posiciones que tracen una dirección sobre cómo se debe actuar, pensar o conducirse. La idea es que generando espacios en donde se converse libremente, se puedan producir múltiples perspectivas, todas ellas dentro de un marco de veracidad, respeto, tolerancia e igualdad de derechos, lo que permite llevar los aprendizajes al plano de la cotidianeidad.

\section{El espacio comunitario}

Una comunidad es un espacio privilegiado de encuentro de saberes cotidianos. Cada comunidad basa su historia en los elementos que se retoman de los conocimientos adquiridos en las relaciones de sus diferentes miembros, en el intercambio de experiencias de todos los que escriben esa historia. La permanencia en el tiempo traduce, sintetiza y orienta los comportamientos de la gente.

Así como la historia de los grupos es importante para sentirse parte de una comunidad, también son importantes las maneras en que esas vivencias se traducen en los sistemas de códigos que denominamos valores culturales; en este caso, se puede decir que esos valores se convierten por inferencia, en valores comunales. Una comunidad puede dar valor a ciertas prácticas que las identifican; el asi somos aqui, traduce simbólicamente este tipo de apropiaciones de las vivencias comunales de un grupo, cuya permanencia en el tiempo y el espacio determina un significado único a esa expresión.

Lo anterior determina, entonces, lo que se hace y lo que no se hace, pero también determina el ser y el deber ser de los grupos de personas. Las comunidades trascienden las colectividades por medio de la interiorización de hábitos que generan costumbres que se traducen en valores culturales estructurantes de procesos que están más allá de esas colectividades primigenias. Es reconocer que antes de que el uno y el otro existan para sí mismos, debe existir el uno para el otro.

Cada comunidad es una cultura, es texto y encuentro. La comunidad es pública, porque su lenguaje lo es; es comunitaria porque su significado lo es. Cada comunidad escribe su particularidad en un texto de encuentro de saberes, de palabras, abrazos, contextos y silencios.

En la estructuración de los grupos a través del tiempo y la permanencia, se dibujan en las comunidades los diferentes actores sociales. Estos actores pueden tener diversas características; sin embargo, todos confluyen en un interés superior, el de la comunidad. Estos actores se pueden determinar de acuerdo con los grupos de edad, con diversas afinidades, con las diferentes 
categorías en las estructuras de poder que se encuentran en el interior de la comunidad, con respecto a los diferentes sistemas de creencias que se desarrollan en las comunidades o con los distintos niveles de participación y de organización comunitaria, entre otros. No obstante, siempre serán aquellos que no saben nada de la economía del dólar, pero una buena mañana se despiertan con un TLC en las alforjas; están atravesados por las estructuras de jerarquía que se desarrollan en cada una de las comunidades de manera autónoma y recurrente.

Entonces, en cada cultura, se encuentran los referentes simbólicos que establecen los vínculos de intercambio entre las personas que de ella participan, códigos que se traducen en el lenguaje por el recurso de la conversación; en ella se gestan los mecanismos de socialización en la que se transmiten los valores, los estereotipos, los papeles sociales, los proyectos de vida y las capacidades de cambiarlos, modificándolos para ajustarlos a las situaciones estructurantes de la cotidianeidad.

En consecuencia, el uso del lenguaje convierte la conversación en un estado emocional de mutua representación interna, mediante la cual el sujeto se permite transformar su marco conceptual referencial y operativo, desde donde toma decisiones frente a situaciones concretas relacionadas con su vida cotidiana. En otras palabras, en la conversación se efectúa una decodificación de la vida cotidiana, la cual no se vive, sino por la representación codificada de los elementos estructurales y estructurantes que la componen, de acuerdo con una esfera de representaciones propias de un espacio y tiempo determinados, siendo la sexualidad de los hombres y las mujeres parte de esta construcción social.

\section{Las Jornadas}

Con estas premisas de trabajo comunitario, nos dedicamos a crear una metodología que incorporara el aspecto comunitario, los aprendizajes sobre sexualidad para todo el mundo y las relaciones entre pares e intergeneracionales. El resultado de la experiencia fue el siguiente:

Con esta estrategia, se potenciaba la capacidad de aprendizaje de los vecinos y las vecinas de la comunidad en torno a los espacios de aprendizaje comunitario, es decir, el sujeto aprendiendo por sí mismo, desde el empoderamiento de las capacidades de aprender conversando, observando y compartiendo sus propias vivencias. 
En este sentido, el concepto de participantes en un proceso de aprendizaje informal se concreta en un proceso de reflexión grupal que implica, en cada uno de los sujetos, un proceso de autorreflexión: condición necesaria para un proceso de aprendizaje en sexualidad conducente a un fortalecimiento de la capacidad de decidir.

A partir de este principio, las Jornadas se enmarcan dentro de la educación no formal, donde las personas aprenden en situaciones de su vida cotidiana, sin la presencia de un educador que guíe su proceso de aprendizaje. Este tipo de aprendizaje, ligado a las estrategias formales y no formales, orienta el aprendizaje hacia la posibilidad que las personas puedan tomar decisiones libres, informadas y responsables en relación con su sexualidad integral.

Así, la Jornada será una actividad de movilización social que permitirá fortalecer los espacios comunales, reconociendo las fortalezas de la autogestión y estableciendo -por este mismo reconocimiento- los niveles de exigibilidad social de las comunidades, por medio de una rica discusión y sensibilización comunitaria en torno a temas que son de interés de las personas que comparten un espacio y una historia; en especial, se visualizan las personas adolescentes como aquellas con más necesidades de atención y de incorporación en la vida comunitaria.

El lenguaje es el elemento dinamizador de las Jornadas, en él se concentra la estrategia de empoderamiento, de autogestión y de exigibilidad de este modelo. En otras palabras, por medio de este recurso, las personas pueden adueñarse -a partir de una demanda- de sus propias dinámicas comunales; de la socialización de estas dinámicas, en donde se comparten los saberes cotidianos, las diversas visiones de mundo según las diferentes generaciones o si son hombres o mujeres, se pueden generar procesos de autogestión que procuren satisfacer dichas demandas. La autogestión constituye un factor para el éxito de las actividades y una instancia adicional de aprendizaje.

Por lo tanto, la comunidad es un espacio donde los vecinos y las vecinas pueden superar los obstáculos para conversar sobre temas que son dedicados más a la esfera privada. De manera tal que se posibilita la transformación cultural y se revitaliza la posibilidad de una construcción conjunta del conocimiento menos caótico, más crítico y más sano.

Este procedimiento permite que todos los sectores de la comunidad interactúen, desde su lugar, en la discusión de tal manera que garantice la retroalimentación de saberes desde diferentes posiciones. Por ello, se torna indispensable la participación de todas las personas: profesionales en salud, 
maestros y maestras, líderes comunales, religiosos y religiosas, políticos y adolescentes en general. Con esto se asegura una verdadera representación de los saberes comunalmente construidos que afirme que los productos de las conversaciones atestiguan una verdadera legitimidad social.

A este proceso se le llama aprendizaje comunitario, pues intenta una movilización social dirigida a romper con un silencio instaurado en torno a la sexualidad, especialmente en una etapa de la vida como es la niñez y la adolescencia, en la cual la prohibición y censura han sido tradición. El ámbito de acción de este programa se establece justamente en la necesidad de transformar los contenidos en la producción simbólica de la cultura, al igual que en sus prácticas políticas excluyentes.

Existe un valor importante que se trata de resaltar en este programa y es el de autonomía como un valor personal a ser promovido desde la niñez y adolescencia. Esto apela a la confianza de que es posible y necesario construir en la adolescencia la capacidad moral pará tomar decisiones importantes en sus vidas, y que los aspectos sagrados, como la sexualidad, son verbalizables, argumentables y dialogables. Incluso se constituye en una necesidad básica en una etapa de la vida marcada por múltiples interrogantes e incertidumbres que conducen a la conformación de una identidad y una conciencia propias. En el desarrollo de su sexualidad, las personas adolescentes ponen en juego mucho más que su cuerpo, el aprendizaje del sexo significa la posibilidad de expresar su capacidad de dar y recibir amor, la definición de su propia identidad y la imagen y valoración de sí mismo o de sí misma.

\section{La estrategia operativa}

Las Jornadas Comunitarias Amor Joven poseen una estructura organizativa que busca contar con la participación y representación de distintos actores. Para ello, se deben conformar grupos de trabajo que contemplen a las instituciones de apoyo en su componente de participación, y a las instituciones públicas y/o privadas, organizaciones locales, profesionales, líderes comunales, padres y madres de familia, representantes de la iglesia y a los y las adolescentes de las comunidades.

Estos grupos se dividen en dos aspectos: los encargados de la preparación, ejecución y seguimiento de las Jornadas, a los que llamamos Equipos Locales, y aquellos que acompañan a los primeros durante todo el proceso; estos últimos son los Equipos de Coordinación y Equipos Técnicos que vienen a ser un apoyo institucional-gubernamental. 


\section{EQUIPOS DE TRABAJO DE LAS JORNADAS COMUNITARIAS AMOR JOVEN}
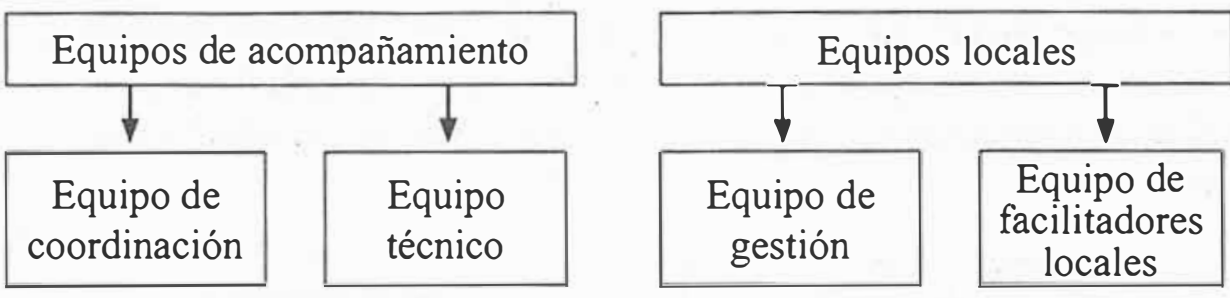

\section{Equipo de coordinación}

Este equipo es el responsable de la coordinación técnica de las Jornadas Comunitarias Amor Joven, debe estar conformado por un representante de la Secretaría Técnica del Programa Amor Joven y otra u otro representante de cada una de las instituciones participantes en el proceso.

Dar seguimiento y apoyo técnico al proceso de las Jomadas Comunitarias Amor Joven

Designar y apoyar las acciones del Equipo Técnico
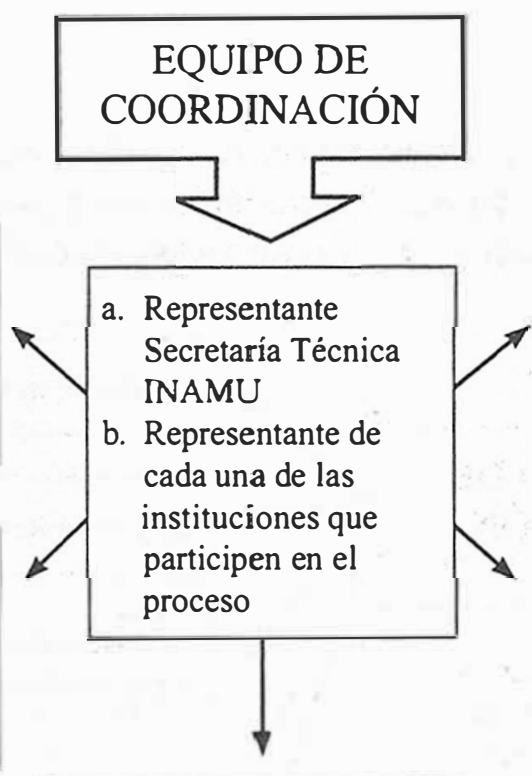

Coordinar la definición de comunidades en las que se realizarán las Jomadas Comunitarias Amor Joven.
Asegurar los recursos humanos y materiales necesarios para la organización, ejecución, evaluación y sistematización

Procurar que las Jornadas Comunitarias Amor Joven mantengan vinculación con los otros procesos del Programa Amor Joven, para asegurar asi una coherencia interna y resonancia nacional de sus objetivos. 


\section{Equipo técnico}

El equipo debe estar conformado por profesionales afines a los objetivos y propósitos de las Jornadas, con alguna experiencia y conocimiento en temáticas relacionadas con sexualidad humana y, específicamente, sexualidad adolescente, organización comunitaria y procesos participativos, entre otras.

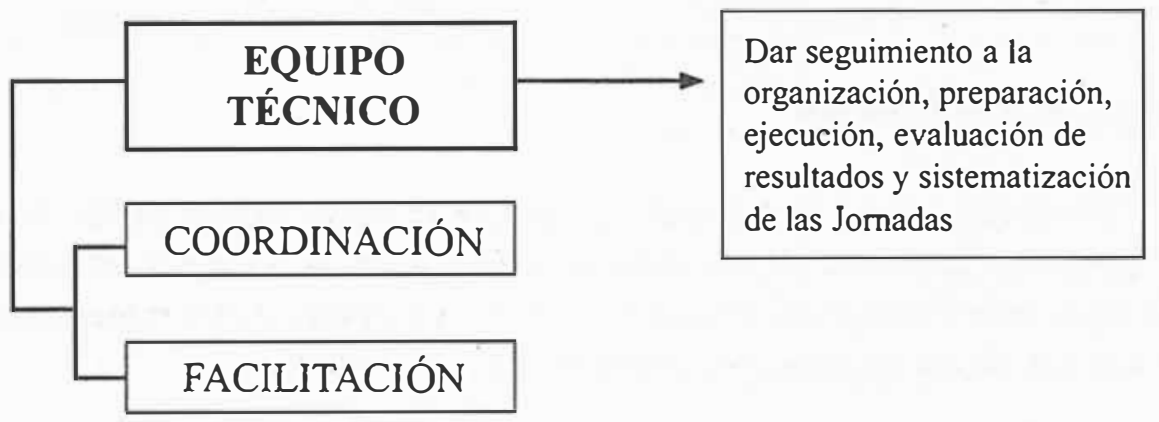

\section{El equipo de gestión}

Se encargaba de todos los aspectos logísticos de las Jornadas, como la coordinación general, la logística, la comunicación y promoción, la convocatoria, la inscripción de facilitadores y observadores locales y participantes.

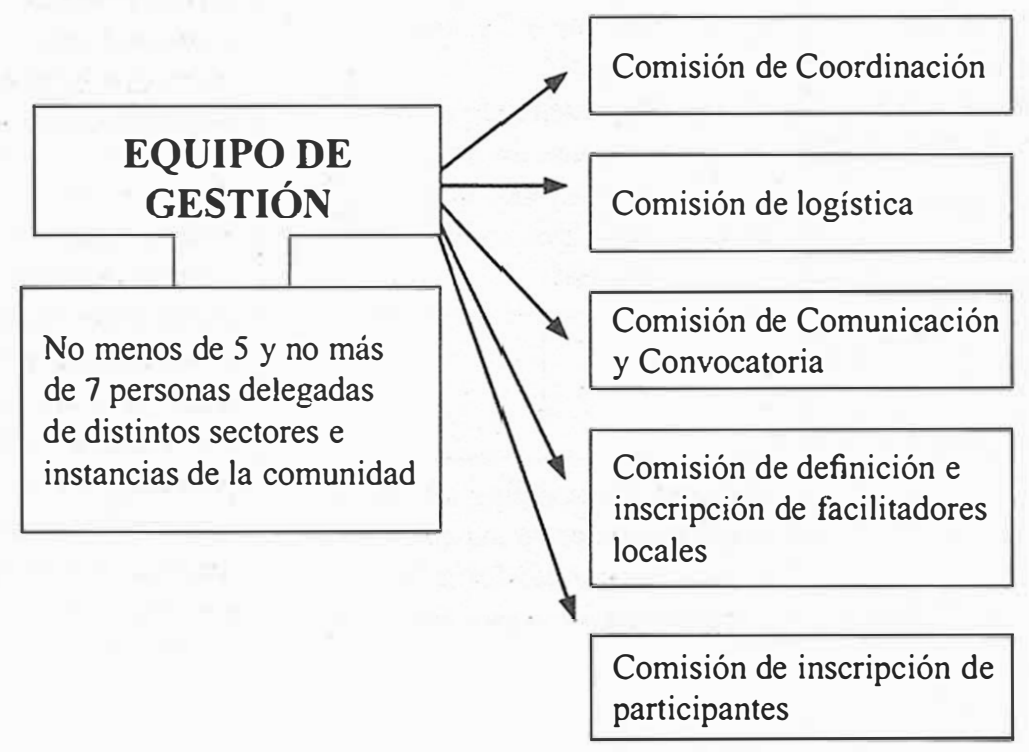




\section{El equipo de facilitadores(as) locales}

Ellos y ellas tienen a cargo los grupos de trabajo en cada Jomada. Estos grupos son de 15 a 20 personas, hombres, mujeres y mixtos; con edades entre los 12 y 15 años, de 16 a 19 años, de 20 a 25 años y más de 26 años.

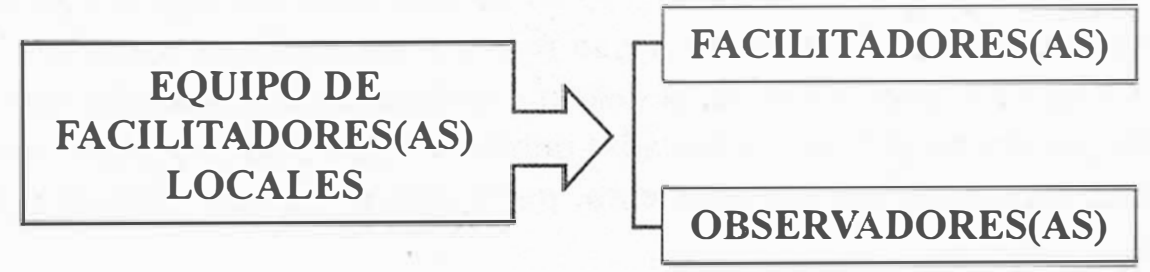

\section{En la jornada}

Con respecto a la estructura lógica de la actividad, esta contempla cinco momentos fundamentales:

- Problematización.

- Información y Orientación.

- Discemimiento y Expresión.

- Evaluación final.

- $\quad$ Proyección para el futuro.

En el momento de la problematización, se busca que en un clima de intimidad grupal las personas expongan y discutan sus principales inquietudes, preguntas, cuestionamientos, experiencias y temores relacionados con la sexualidad adolescente $y$, a partir de esta discusión que integra aspectos racionales y emocionales, se aclaren como grupo posturas e inquietudes que desean los otros conozcan. De alguna forma, este es un paso necesario para la identificación de áreas problemáticas dentro de la comunidad.

En el segundo momento, se promueve el diálogo intergeneracional y entre hombres y mujeres; aquí se busca que exista un encuentro entre las diferentes poblaciones, de tal forma que compartan entre adolescentes y adultos, entre hombres y mujeres, esas inquietudes e interrogantes que han abordado en el primer momento. Asimismo, se quiere que los y las miembros de la comunidad se sientan en la capacidad de construir juntos conocimientos y estrategias para la información y la orientación en el interior de su comunidad. 
Esta es una forma de que la comunidad se apropie de su responsabilidad, sus fortalezas y debilidades en tanto Facilitador y Facilitadora Locales.

El tercer momento se convierte en la posibilidad de que los diferentes grupos de participantes asuman una postura crítica y de denuncia frente a las situaciones, conflictos y problemas ligados a la sexualidad adolescente identificados en el ámbito local.

A pesar de que en el transcurso de la actividad, los equipos locales junto con el Equipo Técnico, se hayan reunido para dialogar sobre la actividad y hacer ajustes menores, el cuarto momento abre una posibilidad de evaluar, en forma global, las Jornadas desde su organización y preparación hasta su ejecución. En este momento, participan el Equipo Técnico y los Equipos Locales.

Para finalizar, el quinto momento contempla las acciones de seguimiento de las acciones comunales posteriores a la Jomada por parte de los Equipos Locales. Aquí participan el Equipo Técnico y los Equipos Locales.

Por otra parte, siguiendo los planteamientos de resonancia comunitaria, la Jornada culminará con una Feria Comunal en la que participen masivamente todos los vecinos y todas las vecinas. De este modo, se pretende amplificar el efecto de resonancia comunitaria de este proceso.

Esta Feria será el punto de encuentro de diferentes estrategias comunitarias; ahí van a confluir y articularse las diversas estrategias del Programa Amor Joven.

Por esta razón, la propuesta parte de la necesidad estratégica de abandonar un enfoque de salud centrado en la oferta (en los proveedores de los servicios de salud) y asumir otro centrado en las personas (en la demanda). Tal como lo sostiene el Programa Nacional de Salud Sexual y Reproductiva de los y las Adolescentes, dependencia del Ministerio de Salud de Brasil (1999), en su documento Espacio joven.

\section{A manera de conclusión}

Es interesante ver cómo las estrategias desarrolladas por el programa en lo institucional fueron permeadas por la lógica informal. También el programa logró introducir y reconocer lo sociable dentro de lo social, demostrando así que ambos espacios permiten la interacción y el mutuo enriquecimiento.

Esto se observa, especialmente, en la estrategia implementada a través del Plan de Capacitación en Educación Sexual desarrollado en el MEP. En la medida en que se abrieron espacios a los docentes, para hablar de sus propias 
vivencias y generar un clima de respeto y escucha, se pudieron generar redes importantes de intercambio y crecimiento personal que incidirán en el desarrollo de la nueva propuesta pedagógica.

Es así como se avanza en el sentido de que el Estado empiece a copiar las formas de aprendizaje de la sociedad y no de imponerle a la sociedad los artefactos innovadores del Estado. Con ello se camina hacia una flexibilización de lo institucionalizado y permite el encuentro entre lógicas diferentes.

El gran reto, sin embargo, es lograr transitar por la lógica de la institucionalidad sin restringir la espontaneidad de esta propuesta dialógica; pues existe siempre el riesgo de que la educación de la sexualidad en el interior de una institución, se transforme en impartir contenidos normados donde las respuestas a los interrogantes estén cerradas y no abiertas a la duda y a la construcción conjunta.

Con lo anterior, se intenta destacar que el Programa Amor Joven logra operacionalizar una estrategia coherente para lograr el objetivo propuesto: la transformación cultural. Y que el aporte es claro en el desarrollo de metodologías concretas que inciden directamente en las prácticas políticas, ya que logra crear dispositivos claros de democratización del saber y construcción colectiva de relaciones más equitativas. 


\section{REFERENCIA}

Ministerio de Salud. (1999). Espacio joven. Programa Nacional de Salud Sexual y Reproductiva de los y las Adolescentes, Marco para el Desarrollo de Experiencias Demostrativas. Brasil. 